\title{
Producing alternating gait on uncoupled feline hindlimbs: muscular unloading rule on a biomimetic robot
}

\author{
ANDRE ROSENDO, SHOGO NAKATSU, KENICHI NARIOKA and KOH HOSODA \\ Osaka University
}

Studies on decerebrate walking cats have shown that phase transition is strongly related to muscular sensory signals at limbs. To further investigate the role of such signals terminating the stance phase, we developed a biomimetic feline platform. Adopting link lengths and moment arms from an Acinonyx jubatus, we built a pair of hindlimbs connected to a hindquarter and attached it to a sliding strut, simulating solid forelimbs. Artificial pneumatic muscles simulate biological muscles through a control method based on EMG signals from walking cats (Felis catus). Using the bio-inspired muscular unloading rule, where a decreasing ground reaction force triggers phase transition, stable walking on a treadmill was achieved. Finally, an alternating gait is possible using the unloading rule, withstanding disturbances and systematic muscular changes, not only contributing to our understanding on how cats may walk, but also helping develop better legged robots.

\section{INTRODUCTION}

Animal locomotion vastly outperforms any other human created locomotion method when it comes to adaptivity. Cats, dogs, horses and even humans can walk in many different conditions, keeping the same gait even at minor obstacles and random disturbances. The mechanisms for this higher stability are not fully understood, but scientists agree that a complex control method (brain) combined with a structure capable of adapting to unexpected disturbances (body) are two fundamental pieces in this conundrum.

Many researchers in the artificial intelligence field try to recreate intelligence with bio-inspired algorithms (e.g. neural networks, genetic algorithms, or refer [1] for more). However, irony abounds by the fact that the number of researchers trying to recreate legged locomotion with a true biomimetic morphology is extremely low. Apparently, conducting computer simulations has more controllable parameters than changing a morphology in contact with real environment, rich in noise and other disturbance sources. Hence, to close the gap between body and brain a better knowledge on the less explored biomorphology would be needed, and as a consequence, robotic locomotion could be improved.

Cats, from tiny domestic cats to big tigers, have baffled scientists over years. Although smaller than most of the dogs, any average domestic cat can climb trees, jump between wardrobes or run faster

The authors acknouledge the Japanese Research Grant KAKENHI Kiban 23220004 and 25540117.

Rosendo, A., Nakatsu, S., Narioka, K., and Hosoda, K. (2014). Producing alternating gait on uncoupled feline hindlimbs: muscular unloading rule on a biomimetic robot. Advanced Robotics, 28(6), 351-365.

Permission to make digital or hard copies of part or all of this work for personal or classroom use is granted without fee provided that copies are not made or distributed for profit or commercial advantage and that copies show this notice on the first page or initial screen of a display along with the full citation. than their canine counterparts. Since this difference can not be attributed to higher computational power, considering smaller brain of cats, we are led to believe that the morphological difference between animals account for their higher specialization interacting with the environment.

Biologists have studied feline locomotion for many years: Engberg and Lundberg [2] studied EMG signals during unrestrained locomotion in cat hindlimbs, while Goslow Jr. et al.[3] identified joint angle and muscle length variations during the same. Herzog et al.[4;5] explores the roles of mono and bi articular muscles at the ankle joint and Wilson et al. [7; 6] compares dissected forelimbs and hindlimbs from cheetahs and greyhounds, aiming to understand differences which could generate a nearly twofold maximum speed difference.

Beyond a muscular level, where only control outputs are observable, biologists hypothesize that the stepping cycle is regulated by afferent signals from peripheral sensory receptors, as stated by Pearson et al.[8]. The idea of sensors generating electromyographic (EMG) signals dates back to 1970, where Severin [9] proved that gamma activation of muscle spindles accounted for $50 \%$ of ankle extensors activity. The inputs for stable gait were well explained with decerebrate cats, where in [10] the absence of ground support reduced muscle activity in $70 \%$, being fully restored when artificially loading the proprioceptors from extensor muscles. This have led scientists to believe that during stance phase more than half of the motoneuron inputs are due to afferent feedback, meaning that unloading the ankle is a necessary condition to start the swing.

With a vast amount of observed data on muscular pattern, joint angles and sensory feedback in cats, one may wonder why robotic performance is still so poor. Although there is a high degree of selfstability on musculoskeletal structures, only a few researchers try to mimic it. Among those trying to better understand animal locomotion replicating it, we could mention Yakovenko [11], which in 2004 developed a simulation with two walking hindlimbs supported by a linear constraint, with 6 muscles per limb. In 2005, Ekeberg [12] provides a groundbreaking simulation with 7 muscles per limb, 2 hindlimbs and a sliding strut as forelimbs, providing stable alternating locomotion while solely using the afferent based muscle unloading rule.

Real world implementations of such approaches are still rare: In 2006 and 2008, Quinn [13] and Tsujita [14] debut a pneumatic artificial muscle based quadruped robot with monoarticular muscles between joints, while in 2011 Hoffmann [15] and Kuniyoshi [16] were more through, also considering contributions from biarticular muscles, famous for their role on energy transfer between joints [17]. Although these four robots explored muscular contributions, no considerations regarding sensorial unloading feedback were taken. On the opposite side of this sphere we can cite Maufroy's work [18], where the sensorial feedback was considered with no regards for the muscular structure, using servo-motors to produce joint torque.

Aiming to shed some light on biological locomotion the proposed rule is tested on a biomimetic robot, which adopts realistic 
link lengths, moment arms and significant muscles for locomotion. To replicate the biological muscle behavior air muscles were used, totalizing 7 active and 1 passive muscle per hindlimb. Similarly to Ekeberg [12], 2 hindlimbs are attached to a sliding strut and tested in a treadmill environment. While at Ekeberg the chosen animal was a domestic cat (Felis catus), in this work we choose as musculoskeletal model a cheetah (Acinonyx jubatus), in face of the little that is known regarding its locomotion.

The robot is capable of walking stably on the treadmill with a simple finite state programming using EMG data from cats to generate a muscular activation pattern. The only feedback source, a small force sensor on its foot, is responsible for stance-to-swing and swing-to-stance transitions. The unloading rule is adopted and successfully generates an alternating gait between hindlimbs, not requiring any kind of coupling between legs to maintain rhythmic behavior. Being the first real world biomimetic implementation of such biological rule, this same concept could help in the future on the development of more stable biped and quadruped robots. Differently from Ekeberg and Maufroy's works [12] [18], different force thresholds and muscle contribution to stability are compared, going beyond just bringing Ekeberg's simulation to real life.

While in this first section we introduce the problem to be approached, section 2 explains the adopted methods, with design, construction and programming details. The third section describes the experiments adopted to validate our robotic stability, while in sections 4 and 5 the results are shown and discussed, respectively. Lastly, we conclude this work in section 6.

\section{METHODS}

The main purpose of recreating a biological system is to perform experiments which can not be performed on the real animal. However, to assure that the performed experiment has any scientifical significance the amount of reproduced features must be great enough to replicate intended phenomena. This way, we focused on replicating the musculoskeletal system with force sensing capability, and no special attention was paid to other features (vision, fur, etc). Due to the low speed, the construction of the tail was also ignored.

\subsection{Hindlimbs design}

In [19], a comparison among members of the Felidae family is drawn, showing locomotion and morphologic similarities with felines ranging from 4 to $200 \mathrm{~kg}$. Adopting link lengths from this work, combined with musculoskeletal information from [6], we opted for the creation of a 3-link hindlimb (femur, tibia and metatarsus) with 3 degrees of freedom and 8 flexion/extension muscles (as previously approached in [20]). These hindlimbs are attached to a hindquarter, which are fixed to a sliding strut to simulate a forelimbs-spine assembly. Although resembling a cheetah in structure, for aesthetic reasons we preferred to name it as a member of the Panthera pardus species, hence called Pneupard. A picture and CAD design image of the same can be found in Figs. 1 and 2.

From the 8 available muscles for flexion/extension, five are monoarticulars and three are biarticulars. The monoarticular muscles are biceps femoris (hip extension), iliopsoas (hip flexion), vastus lateralis (knee extension), soleus (ankle extension) and tibialis anterior (ankle flexion). All of them active muscles, with the exception of tibialis anterior. Although biceps femoris is not a monoarticular muscle per se, the small size of its moment arm around the knee directed us to a simplification, approximating it to zero.

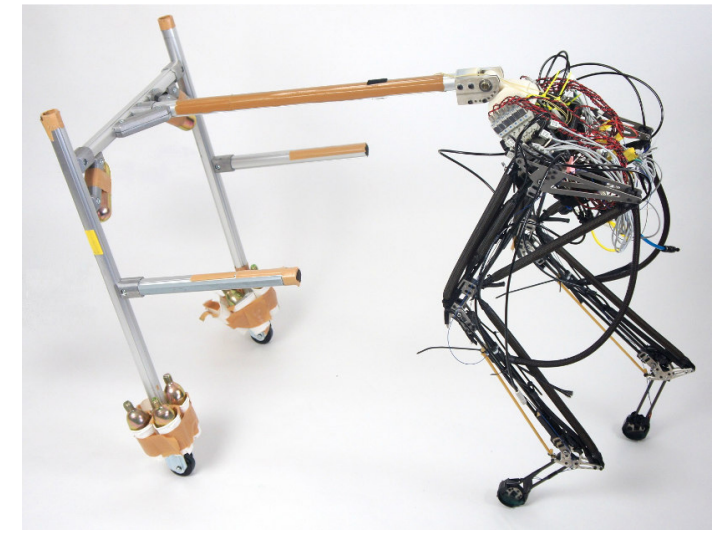

Fig. 1. The robot possess hindlimb dimensions similar to a cheetah, with realistic moment arms to produce scientifically relevant locomotion during EMG-based muscular activation.

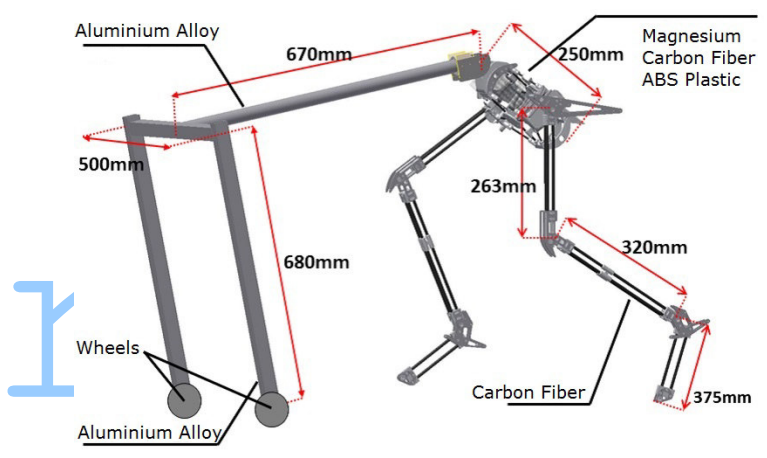

Fig. 2. CAD design of Pneupard with important measurements depicted.

The remaining three biarticular muscles are semitendinosus (hip extension and knee flexion), rectus femoris (hip flexion and knee extension) and gastrocnemius (knee flexion and ankle extension). Gastrocnemius and rectus femoris are known for transfering force between joints, according to [17]. Semitendinosus, on the other hand, has a flexor characteristic, as observed by Goslow [3], being solely used during phase transitions. Two additional muscles are fixed throughout the experiments, constraining adduction-abduction movements with a certain degree of compliance. If needed, movements of adduction (passive) or abduction (active) can be performed, imitating muscles such as gracilis, caudofemoralis or pectineus. A table with overall muscle lengths, adopted moment arms, nomenclature and articulation type can be found at Table I.

Aiming to provide a high fidelity between robot and animal, origin and insertion points were maintained, respecting muscular moment arms. A single exception was made during the attachment of the muscle iliopsoas, where in the animal it has origins on the animal's trunk, attaching on the proximal part of the femur. In our robot, we had to attach it in the distal part of the femur to share the same attachment platform as the other hip muscles. In Fig. 3 a schematic drawing represents the adopted muscles origin and insertion points. 
Table I. Pneupard's muscular specification

\begin{tabular}{lccc}
\hline Muscle & Length[mm] & Max. Moment Arm[mm] & Articulation type \\
\hline Soleus(SO) & 170 & $61($ ankle) & Monoarticular \\
Gastrocnemius(GA) & 390 & 41 (knee), 71(ankle) & Biarticular \\
Vastus Lateralis(VL) & 145 & $27(\mathrm{knee})$ & Monoarticular \\
Semitendinosus(ST) & 440 & $135(\mathrm{hip}), 102(\mathrm{knee})$ & Biarticular \\
Biceps Femoris(BF) & 320 & $150(\mathrm{hip})$ & Monoarticular \\
Rectus Femoris(RF) & 330 & 47 (hip), 22(knee) & Biarticular \\
Iliopsoas(IL) & 230 & $47(\mathrm{hip})$ & Monoarticular \\
Tibialis Anterior(TA) & 230 & $48($ ankle) & Monoarticular \\
\hline
\end{tabular}

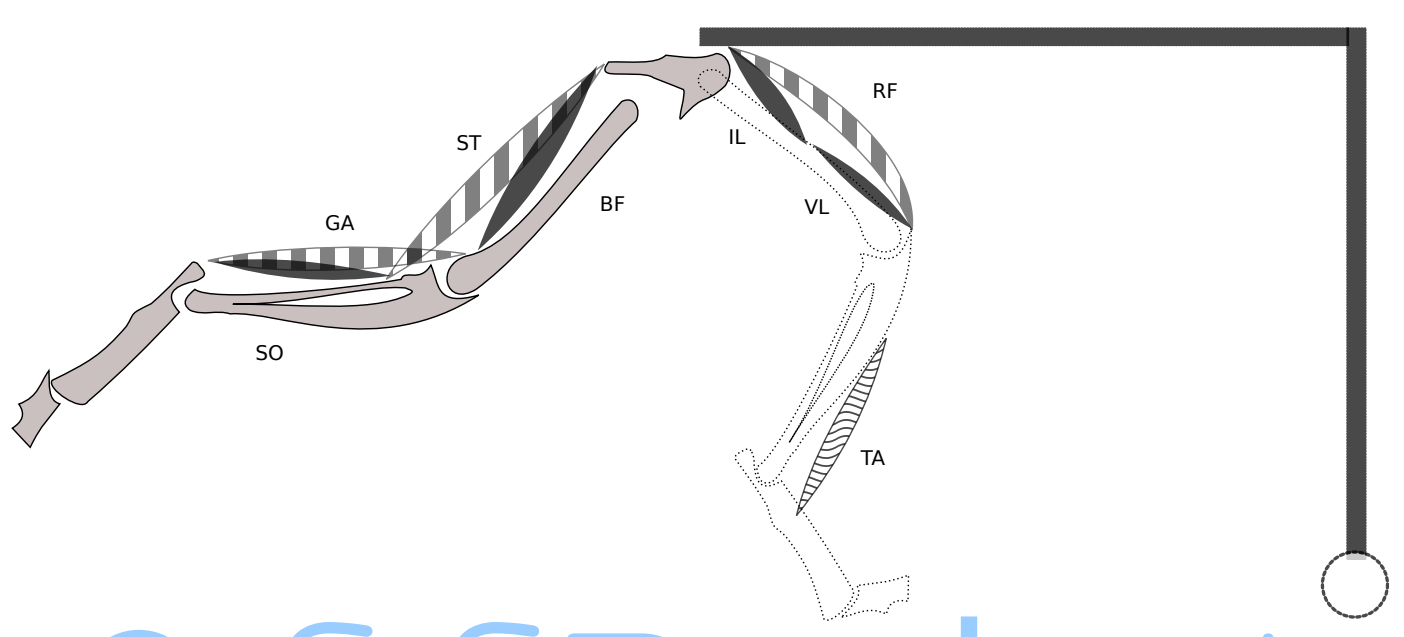

Fig. 3. Diagram with Pneupard's musculoskeletal structure. Muscles in solid black are monoarticular muscles, while muscles in thick stripes are biarticulars. Right and left hindlimbs have different colors, passive muscles have a thin striped pattern and muscles related to adduction and abduction are not depicted on this diagram.

\subsection{Muscular consideration}

While animals possess biological muscles, which provide actuation combined with compliance to interact with the environment, the same so far is not available for human-created structures. Alternatives such as series elastic actuators [21] came into play for almost 20 years, but the amount of weight added to the robot is cumbersome. When it comes to power-to-weight ratio, actuators such as air muscles outperform any other alternative, reaching ratios as high as 400:1, being successfully used in robotic applications [22].

The principle of activation is based on the intake of air through a pneumatic valve, which generates a contraction of the muscle, while exhausting the same air relaxes the muscle. The compliance offered by the actuator is proportional to the muscle contraction, while the force provided by the same is, as mentioned in [23], dependent on the internal pressure and muscle deformation, as shown in the following equation:

$$
F \propto \frac{p_{a i r}}{\Delta l / L_{0}}
$$

where $F$ is the force, $p_{\text {air }}$ is the internal pressure, $L_{0}$ is the relaxed length and $\Delta l$ is the deformation after muscular activation. Comparisons have shown that although force-length properties of these actuators are muscle-like, with higher activation pressures resulting higher forces and longer muscles generating higher outputs than short ones, force does not decrease with increasing contraction speeds [24]. To be considered as a fair replacement it should be used below the resting length (biological muscles degrade performance above this length) and at low contraction speeds, as used during walking experiments. The resemblance between these artificial muscles and biological ones have been exploited by roboticists[25] and biologists[26].

Our actuators are made from a rubber tube with $8 \mathrm{~mm}$ internal diameter and $1 \mathrm{~mm}$ thickness, covered by a polyester exterior braid with minimum diameter of $9 \mathrm{~mm}$ and clamped in both sides with plastic connectors with $8 \mathrm{~mm}$ diameter, where a cap seals one side and a plastic tube provides air through the other.

These muscles are filled with air provided by 14 pilot operated on-off valves. A control method called hysteresis control [20] allows a certain degree of pressure control inside each muscle for different activation levels. The choice of on-off valves over proportional valves is due to the fact that, considering the same weight, it is possible to have higher flow rates with the former, allowing fast movements. Thus, the hindquarter-hindlimbs assembly weighs less than $3 \mathrm{~kg}$ (each hindlimb weighting less than $600 \mathrm{~g}$ ) while having proportions of a cheetah. The maximum pressure used is 0.6 $\mathrm{MPa}$, where our manufacturing process guarantees a life time of a few years, decreasing to months if used above $0.7 \mathrm{MPa}$. Due to our non-industrial manufacturing process, muscle length repeatability varies $5 \mathrm{~mm}$.

Adopting EMG signals from walking cats [2], similarly to Ekeberg's simulation [12], we constructed a finite state control with 4 distinct phases: Lift off, Swing, Touch down and Stance. Each phase has a predefined target pressure for each muscle, and the right and left hindlimbs are uncoupled. The initial phase is Touch down, where the microcontroller samples for activity on the force sensing resistor at the tip of the hindlimb. When stimulated, the 


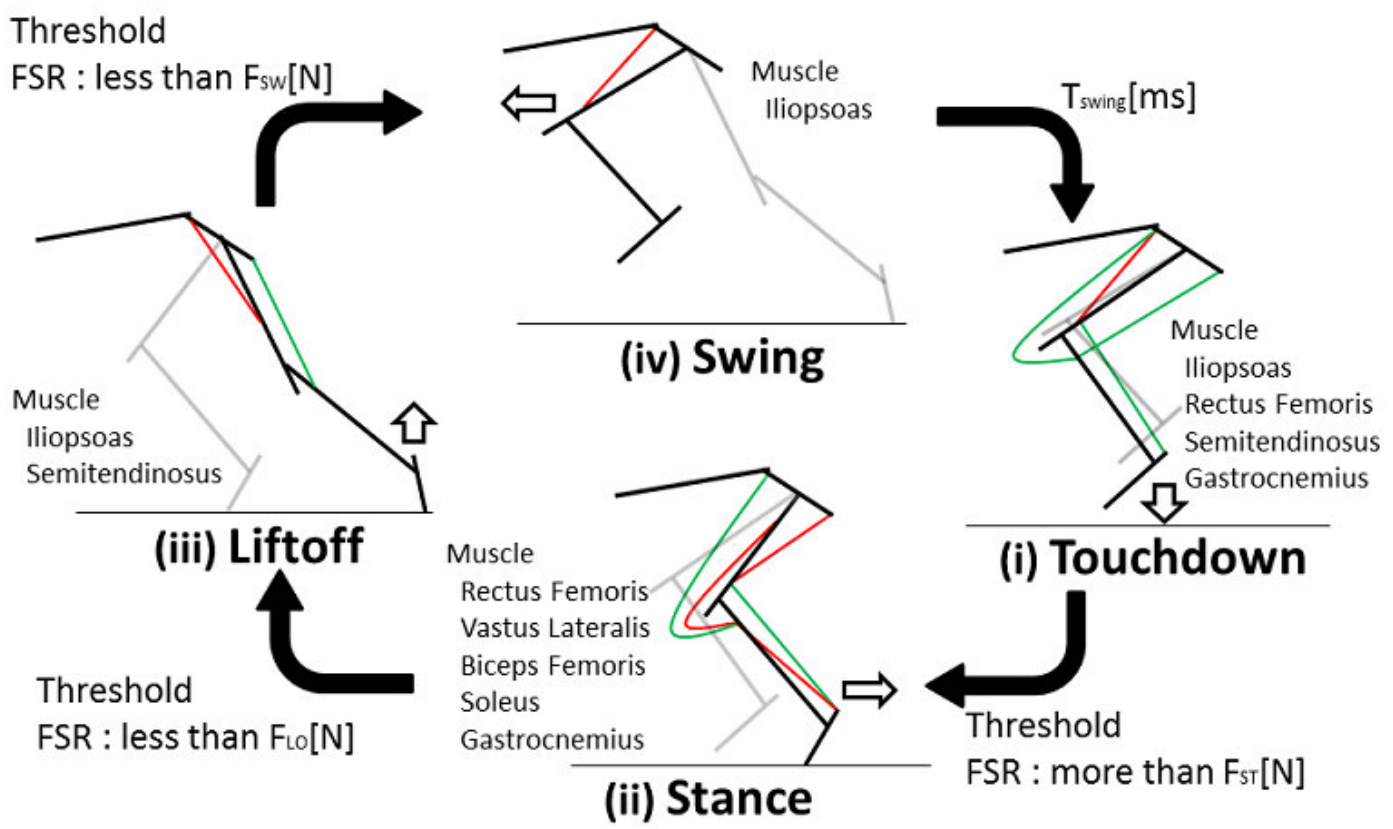

Fig. 4. Four states of the finite state controlling on the hindlimbs. We can see the contribution of each muscle during each phase.
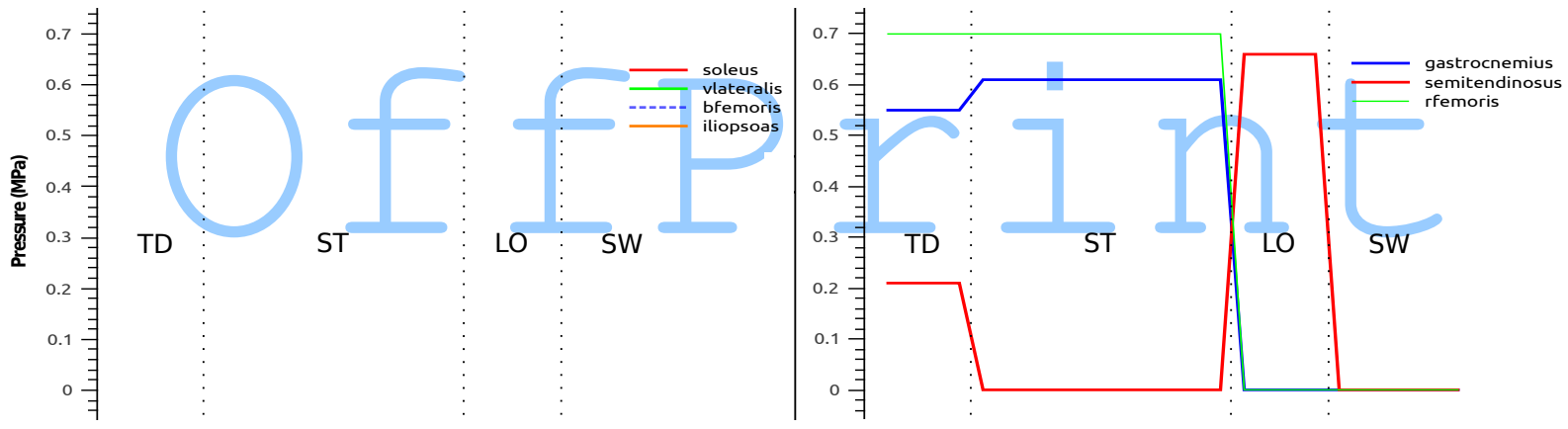

Fig. 5. Activation pattern for the hindlimbs. On the left inset we have monoarticular muscles, while on the right one biarticular muscles are depicted. The four phases of the gait (TD, ST, LO and SW) are defined as touch down, stance, lift off and swing, respectively.

state is switched to Stance, which lasts until the ground reaction force acting on the sensor decreases to a value below a predefined threshold, activating then the Lift off phase. The end of the Lift off phase happens when the contact between the sensor and the floor no longer exist, starting the Swing phase. Differently from Ekeberg, Pneupard's swing phase is solely based on a timer interrupt, not requiring a second sensor on the hip. After the specified time, the hindlimb enters Touch down phase again, waiting for floor contact.

The design choice of not implementing hip angle as a swing limit parameter is based on biological data which proves that swing phase duration has low variance with different velocities (stepping frequency), as shown in [27]. The system could walk stably without this angle information, using a fixed $350 \mathrm{~ms}$ as transition timer. The four states of the adopted control method are depicted in Fig. 4. Since this work aims to prove that the unloading rule is capable of creating a stable locomotion in musculoskeletal systems, other sensors (gyroscope or accelerometer) were not adopted.

The adopted activation pattern for Pneupard is depicted in Fig. 5. Since biological muscles and artificial muscles have different properties, a fine tuning process (described in Experiments section) was required to translate the muscle signal into proper pressure signals. Contributions from higher-level controllers (brain) and lowerlevel controllers, such as spine-generated central pattern generators, were ignored in order to focus on the intended phenomenon of rhythmic gait through unloading rule in decoupled hindlimbs. The coupled hindlimb alternative was not tested, since it was proven in simulation that it did not improve the system behavior during unloading rule [12]. Moreover, coupled hindlimb controls are already broadly studied by the majority of roboticists (e.g. [13] [14]), being the uncoupled control a fairly unexplored field.

While Maufroy [18] approaches unloading rule in uncoupled hindlimbs in his work, the lack of a musculoskeletal compliant structure inhibits it from observing muscular contribution to stability or a deeper study on joint angles and force sensors during such compliant locomotion. Our study simulates muscles, considering compliance during gait and EMG-based muscular activation, mimicking animal locomotion to a higher extent than the former, 
which used pre-defined trajectories on non-compliant electric motors attached to limb joints.

Differently from Ekeberg's simulation, we used a force sensor on each hindlimb, instead of soleus muscular tension, as ground force measuring method. The force sensing resistor (Interlink Electronics, FSR408) was tuned, specially considering the sensor's precision, degraded by deformable materials on the tip of the hindlimb.

Preliminary experiments with pressure sensors on the soleus muscle have shown that during stance phase the noise created while activating other muscles resulted in accidental triggering of the unloading rule. The addition of a low-pass filter degraded the system response, and other equally sensitive alternatives (such as strain gauges) are likely to suffer from the same shortcoming. In the future, we will adopt a muscle tension measuring alternative to better reproduce this biological idea.

\section{EXPERIMENTS}

Experiments were conducted in a controlled environment, with a treadmill running at constant speed $\left(0.8 \mathrm{~km} \mathrm{~h}^{-1}\right)$ and a tether connected to the robot, supplying energy $(12 \mathrm{~V})$, compressed air $(0.6$ $\mathrm{MPa}$ ) and exchanging robot attitude data with a computer (muscle pressure, force sensor signal). With the sole purpose of understanding and reproducing feline locomotion on a treadmill, no special attention was paid to energy efficiency, air consumption or remote applications.

The treadmill speed was defined after trials with the robot, being the chosen speed the robot's baseline speed. Initially, Ekeberg's adopted activation pattern [12] and EMG signals were compared, creating an activation pattern inspired on these levels. As this pattern did not create a stable walking, joint angles from videos with walking cats were compared with observed values and specific muscles were tuned at 0.2 bar increments until a roughly stable walking was possible. From this point, an iterative process started, tuning force sensor thresholds and muscle activation pattern to reach the most stable walking, thus called baseline. Aiming to validate the unloading rule on such morphology this trial and error method was chosen over optimization algorithms due to the vast data on cats locomotion, angles and time constraints, where other methods could have created other locomotion gaits, outside of our research scope.

The experiments were conducted in two different experimental settings: a first experiment aimed to understand individual contributions from muscles on the gait stability during cat stepping, while the second introduced obstacles to see what influence it had on the robot rhythmic stability. Both experiments adopted the unloading rule as an alternating phase attractor state, as suggested by Ekeberg [12], and aim to prove that this rule is adaptive enough to withstand morphological (muscle pressure) and environmental (obstacles) changes in a real world experiment, while Ekeberg only considered environmental changes.

As a stability measuring criteria, we registered the influence of these disturbances on the phase difference between right and left hindlimbs. Similarly to Ekeberg, the phase difference is defined by:

$$
\Phi_{H(\text { actual })}=\frac{T_{H(\text { actual })}-T_{O(\text { previous })}}{T_{O(\text { next })}-T_{O(\text { previous })}}
$$

where $\Phi_{H}$ is the specified hindlimb's phase, $H$ and $O$ standing for hindlimb and opposite hindlimb and $T$ standing for the moment in which the hindlimb touches the floor. The idea is that the phase of each hindlimb is defined in association with the floor touching moments of the opposite hindlimb.
While the robot walks on the treadmill, many methods for acquiring data were used: the microcontroller which controls the robot sends attitude serial data to a computer, and on the outside a motion capture system is used to acquire data pertaining to the robot's position, speed and angles.

\subsection{Experimental Method}

The experiment starts by placing the robot on the running treadmill. When the hindlimbs, which are in Touch down phase, touch the treadmill, walking starts naturally. The parameter being tested is set prior to the experiment, adopting the baseline with one variable value, which might be muscular pressure or force sensor threshold, and 20 steps are recorded on the motion capture system. When a stable walking is not possible, with the robot falling before 20 steps, the experiment is labeled as a fail and halted. Second and third trials of unsuccessful experiments didn't result in a success, usually collapsing before reaching a stable 10th step.

Although in the majority of the success cases more than 40 steps were possible, only 20 recorded steps are plotted, with no observable stability difference between the recorded and unrecorded experiments. Falling after long time stable walking happened by the robot falling through one of the extremities of the treadmill (too slow or too fast), breaking parts (many ABS plastic parts fatigued during experiments) or slippage (after starting the stance phase, the leg slips to the back, finishing stance before the opposite leg started it). Failed walking experiments usually happened by lack of muscle strength or incorrect hindlimb coordination.

On the second experiment, baseline parameters are used to measure the alternating gait stability when different obstacles are introduced on the treadmill. Initially, stable gait is produced and then the obstacle is introduced on the treadmill. Experiments are labeled as a success if 6 steps are produced after the obstacle was passed. All experiments labeled as success were capable of continuing walking after 6 steps, but we restricted the plotted data to 6 steps to focus on the gait disturbance phenomenon (being 3 steps on each leg enough to bring the system back to steady state). In Fig. 6 and 7 the robot overcoming an obstacle on the treadmill is shown from a video camera and a motion capture system, respectively.

\section{RESULTS}

Initial trials allowed the reproduction of a stable walking on the treadmill, defining the baseline for the robot. In Fig. 8, knee, ankle and hip angles, extracted with motion capture, are shown during a stable walking on the treadmill. During stepping the angular range of motion for ankle, knee and hip are respectively $17^{\circ}, 50^{\circ}$ and $40^{\circ}$.

Focusing on swing and stance phases, Fig. 9 shows the behavior of the hindlimb during these two phases. The ground clearance produced during swing is of $40 \mathrm{~mm}$, while the stride length is $450 \mathrm{~mm}$. To prove that the alternating gait produced by the unloading rule is stable enough to overcome disturbances we proceeded to tests with different parameters.

\subsection{Systematic changes on walking parameters}

The influence on the alternating stability was initially tested with different threshold values at the ground reaction force sensors, as seen in Fig. 10, and later the same contribution was evaluated for different pressures on gastrocnemius muscle during stance phase (Fig. 11). While small thresholds could still produce stable walking, bigger thresholds greatly decreased stability. For the gastrocnemius muscle, any pressure lower than $0.36 \mathrm{~N}$ was insufficient to 

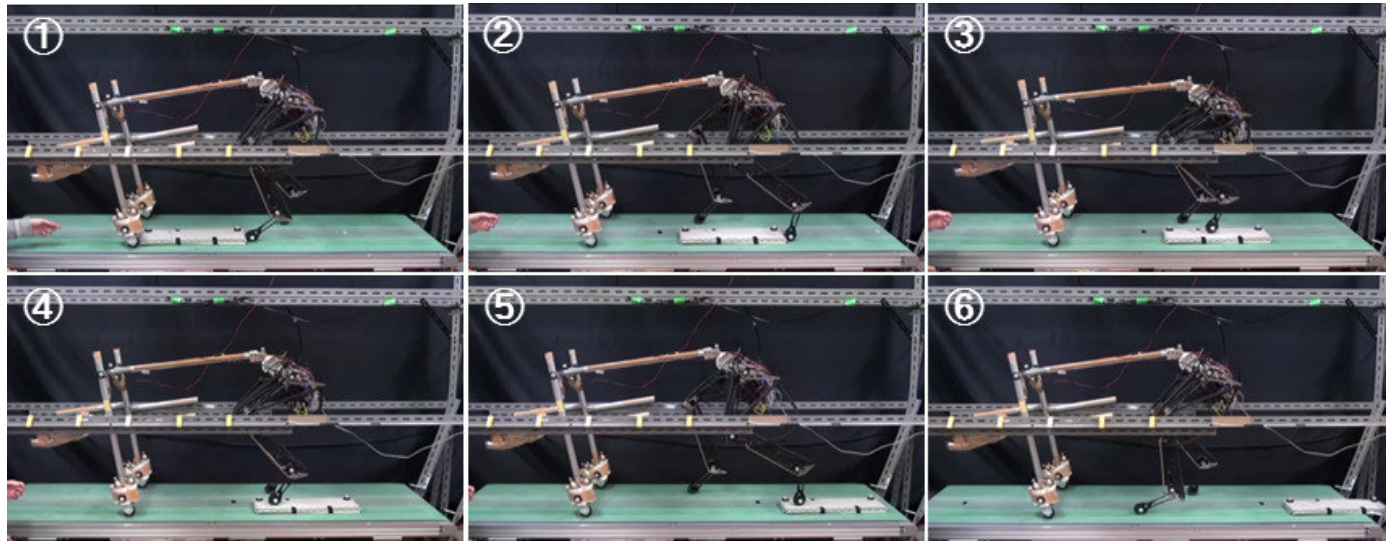

Fig. 6. Walking experiment with hindlimbs attached to moving structure, passing through an obstacle. From the top left to the top right an alternating gait with left stance and then right stance. In (3) the left leg touches the obstacle, initiating stance at (4), continuing the alternating gait afterwards.

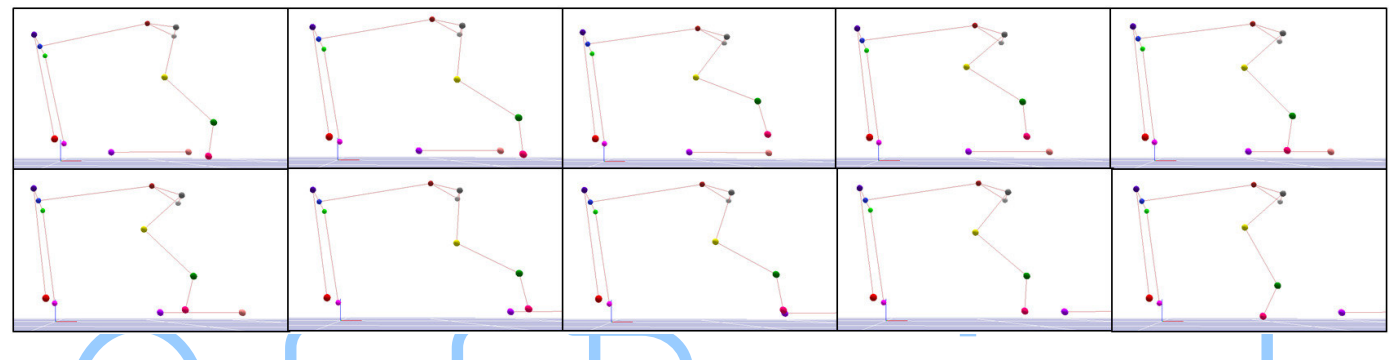

Fig. 7. Walking experiment with an obstacle, recorded by a motion capture system. Markers attached to the left hindlimb show it overcoming an obstacle, extracted from Fig. 6. Movement progresses from top left corner to top right corner, and then from bottom left to bottom right.

keep the robot standing during stance, while bigger pressures also degraded walking stability.

The next step consisted in analyzing contributions from different muscles. Alternating gait stability with the muscle rectus femoris during stance was evaluated, as seen in Fig. 12.

During walking rectus femoris proved to be essential for weight bearing, resulting in body collapse after a few steps whenever the pressure was lower than $0.3 \mathrm{MPa}$. Differently from gastrocnemius, an upper limit for rectus femoris stability during stance was not observed.

\subsection{Systematic changes on walking environment}

A second set of experiments used the baseline setting to measure influence of obstacles on gait stability. Initially we used a motion capture system to analyze the effects of an obstacle on joint angles during walking (similarly to previously shown Figures 6 and 7). In Fig. 13 we can see the hindlimb overcoming an obstacle, while in Fig. 14 the hip, knee and ankle joint angles from this experiment are shown.

Upon finishing touch down phase prematurely, the hindlimbs have to account for the height difference, with the effect being dealt at a morphological level (no change in the activation pattern), without compromising alternating gait. In Fig. 13 the knee (a) and ankle (b) joint behavior during disturbance is emphasized.

Finally, trials with obstacles of different heights were performed on the treadmill, registering the alternating gait stability while overcoming obstacles (Fig. 15). Although the robot could pass through $8 \mathrm{~mm}$ obstacles without major stability disturbances, bigger ob- stacles created bigger disturbances. With a gradually increasing height, eventually the robot was not capable of stabilizing after stepping on an obstacle, collapsing at obstacles with $35 \mathrm{~mm}$.

\section{DISCUSSION}

\subsection{Alternating gait through unloading rule}

Inspired by findings from [12], where an alternating gait could be reproduced solely by using a bio-inspired unloading rule, we developed a real-life experiment with realistic moment arms and adopted the same rule. With the intent to assess if such rule would be capable of generating an alternating gait, initial trials were performed, tuning muscles and sensors. After some tests, the walking gait presented a natural alternating behavior, which prevented the robot from entering a bounding gait condition.

Comparing hip, knee and ankle angles from Fig. 8 to angles extracted from unrestrained animals [3], the three curves from the robot resemble the biological curves, with two noticeable differences: First, the biological ankle range of motion is $30^{\circ}$, which is more than that observed on the robot $\left(17^{\circ}\right)$. Second, the hip swing angular speed on the robot is faster than values observed on cats (steep hip curve pointing downwards in Fig. 8). Although these differences are not really associated with the unloading rule per se, they may imply that even though the movement was slightly unnatural, the attractor state created by the unloading rule compensated for these differences. We believe that further tuning on the baseline parameters to reproduce a more compliant ankle joint and a smoother swing would make the robot behavior more animal-like. 

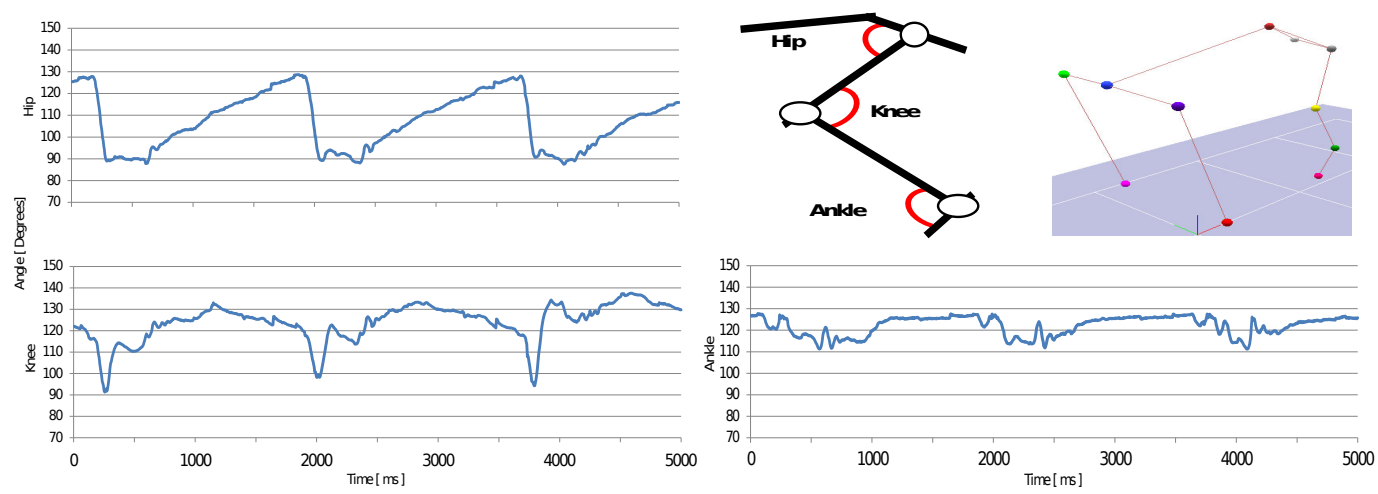

Fig. 8. Angular position of hip, knee and ankle during walking with baseline parameters.

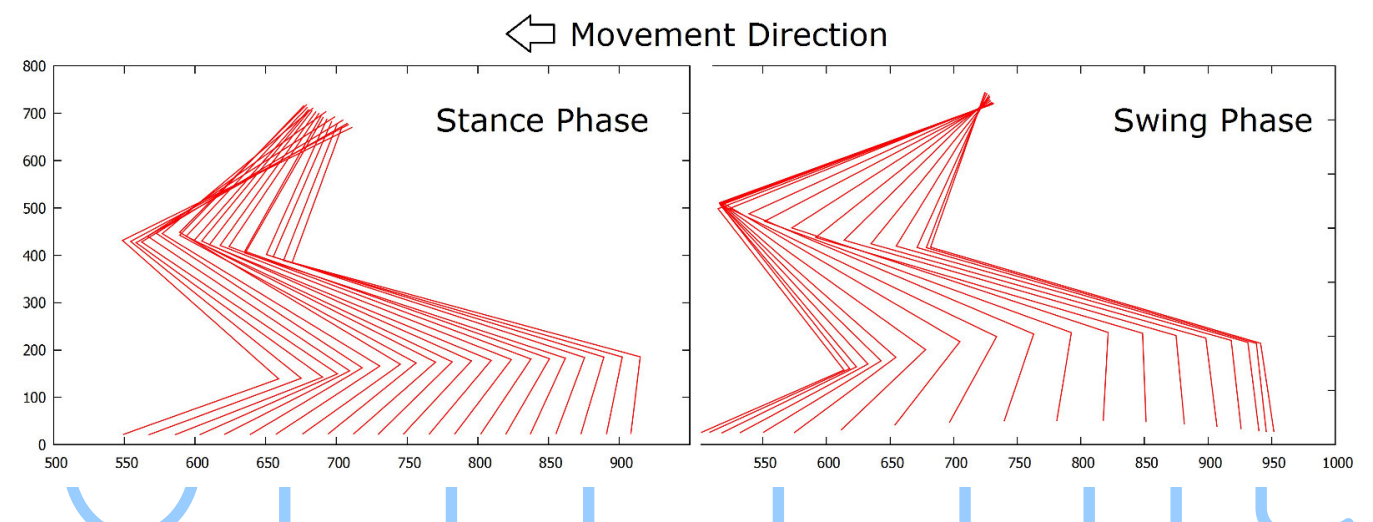

Fig. 9. Digitized stance and swing phases recorded with a motion capture system with baseline parameters. Both stick figures are representative data, based on the mode of 20 steps, having a very low variability between steps. $\mathrm{X}$ and $\mathrm{Y}$ axis are expressed in milimeters.

While in Ekeberg [12] a symmetric gait is observable during unloading rule, the same was not true in our experiments. While in computational environments reproducing perfectly symmetric legs is possible, the errors involved in the artificial muscle construction collaborated to different muscular lengths between legs. Our experiments had a stable gait with one hindlimb's phase at 0.7 and the other at 0.3 , meaning that contracted muscles made one look shorter than the other, even though both have exactly the same length (both hindlimbs were made in high precision equipments, such as industrial grade $\mathrm{CNC}$ and fused deposit modeling $3 \mathrm{D}$ printer). One more time, the fact that the stability was maintained amidst assymetric construction fortifies the hypothesis that the attractor state created by the unloading rule is strong enough to overcome systematic disturbances.

In Fig. 10 different threshold values were used, resulting in stable gait up to a threshold value of $11.8 \mathrm{~N}$. Since stance-to-lift off thresholds are reached when the ground reaction force naturally decreases, higher thresholds forced the robot to shift to lift off before the stance was supposed to finish. In many such occasions, the stance phase was so short that the opposite hindlimb was still in swing, creating a flight phase, which was not supposed to be observed in walking. Consequently, with the opposite hindlimb landing in swing phase (with a different attack angle than the one observed in touch down) the robot collapses.

\subsection{Muscular contribution to alternating gait}

The muscular activation pattern developed during trials served as a template to be repeated, being the unloading rule the trigger for phase changes. Among 7 active muscles used during this experiment, only gastrocnemius and rectus femoris were chosen as variable parameters. The main reason for this choice is that gastrocnemius and rectus femoris are, among with soleus, vastus and biceps femoris, the major weight bearers in animals. Focusing on an ankle extensor and a knee extensor allowed us to see different effects on walking. Future works with this same platform will investigate contribution from other muscles on a walking experiment.

In Fig. 11 experiments with gastrocnemius muscle were shown. Two collapsing situations were clearly depicted: Low gastrocnemius pressure and high gastrocnemius pressure. For the first case, decreasing the amount of force done by gastrocnemius passes the extra burden to soleus, which [6] proved to be more than 4 times smaller than gastrocnemius in a cheetah. This way the robot collapses for lack of ankle stiffness, standing in a 2-link position (plantigrade) instead of a 3-link (digitigrade). The second case results in an overstiff muscle, locking the ankle joint at $130^{\circ}$, not complying when touching the floor. The angle of $130^{\circ}$ was set as a maximum value with a hard mechanical stop for walking experiments. Apparently, compliance plays a very important role on ankle joint, where an overcompliant or overstiff ankle affects stability negatively. 

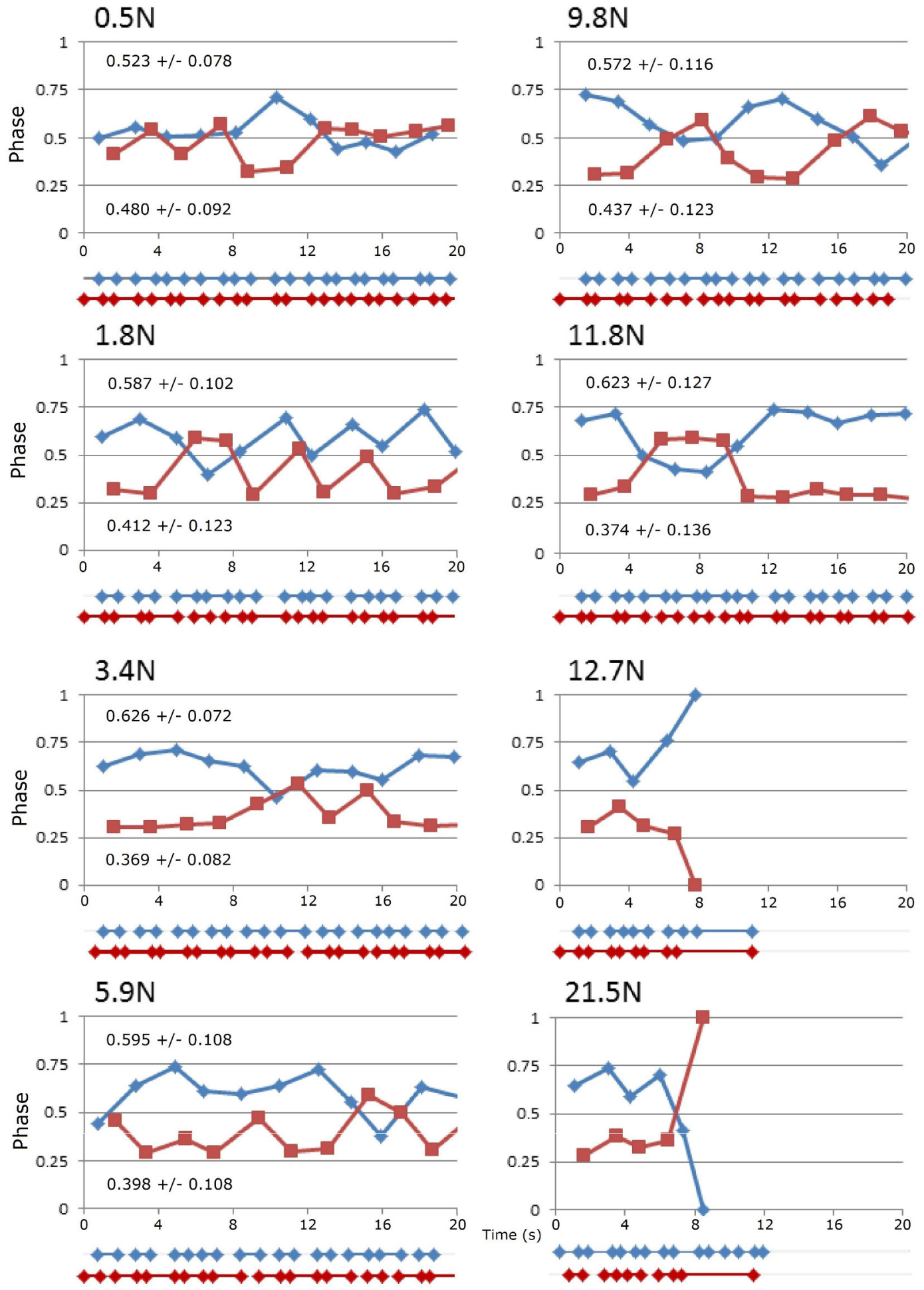

Fig. 10. Contribution to alternating gait for different values of stance-to-swing threshold. Red lines represent right hindlimbs, blue lines represent left hindlimbs and their mean and standard deviation are depicted on each graph. 

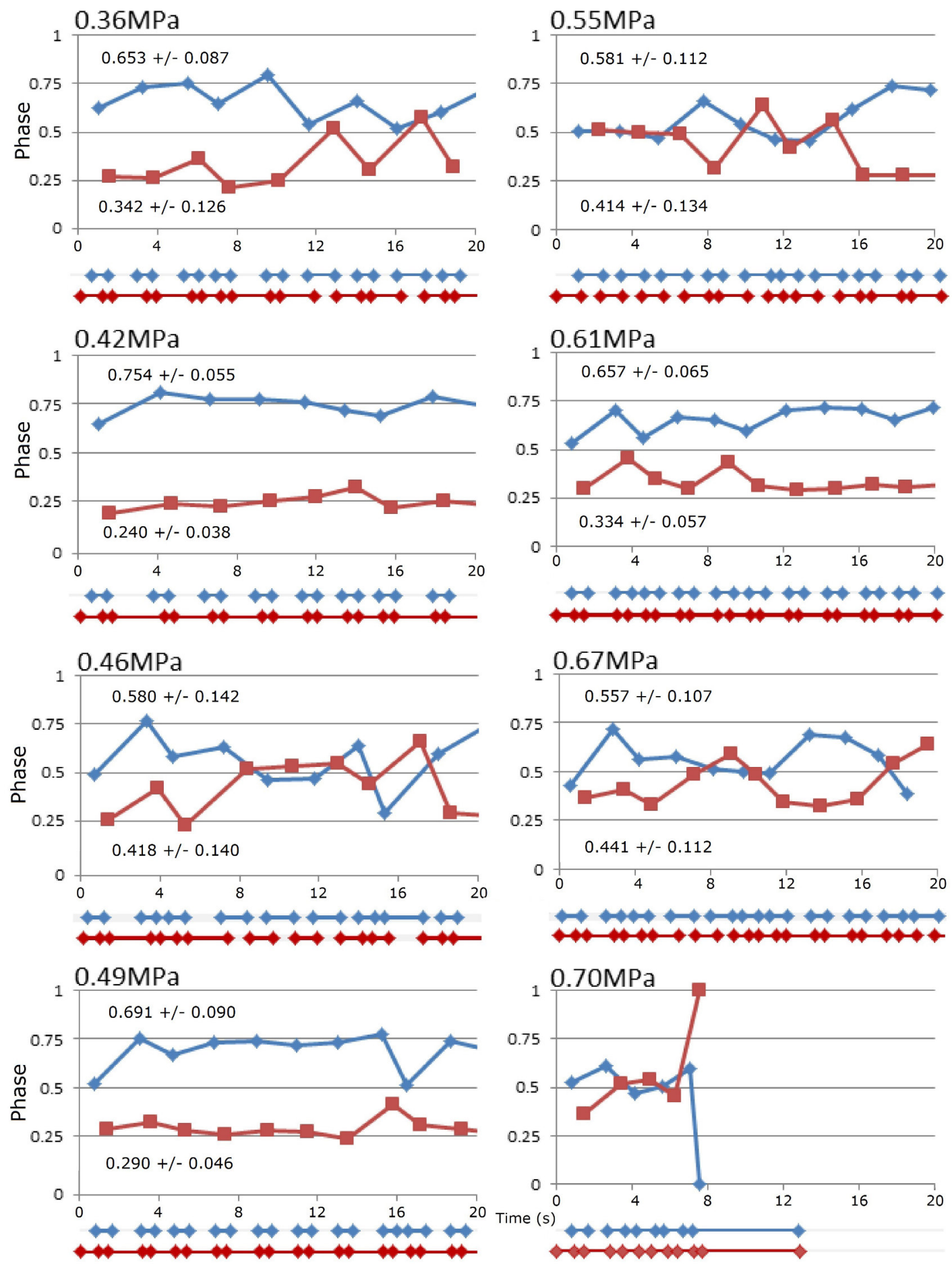

Fig. 11. Contribution to alternating gait for different values of gastrocnemius pressure during stance. Red lines represent right hindlimbs, blue lines represent left hindlimbs and their mean and standard deviation are depicted on each graph 

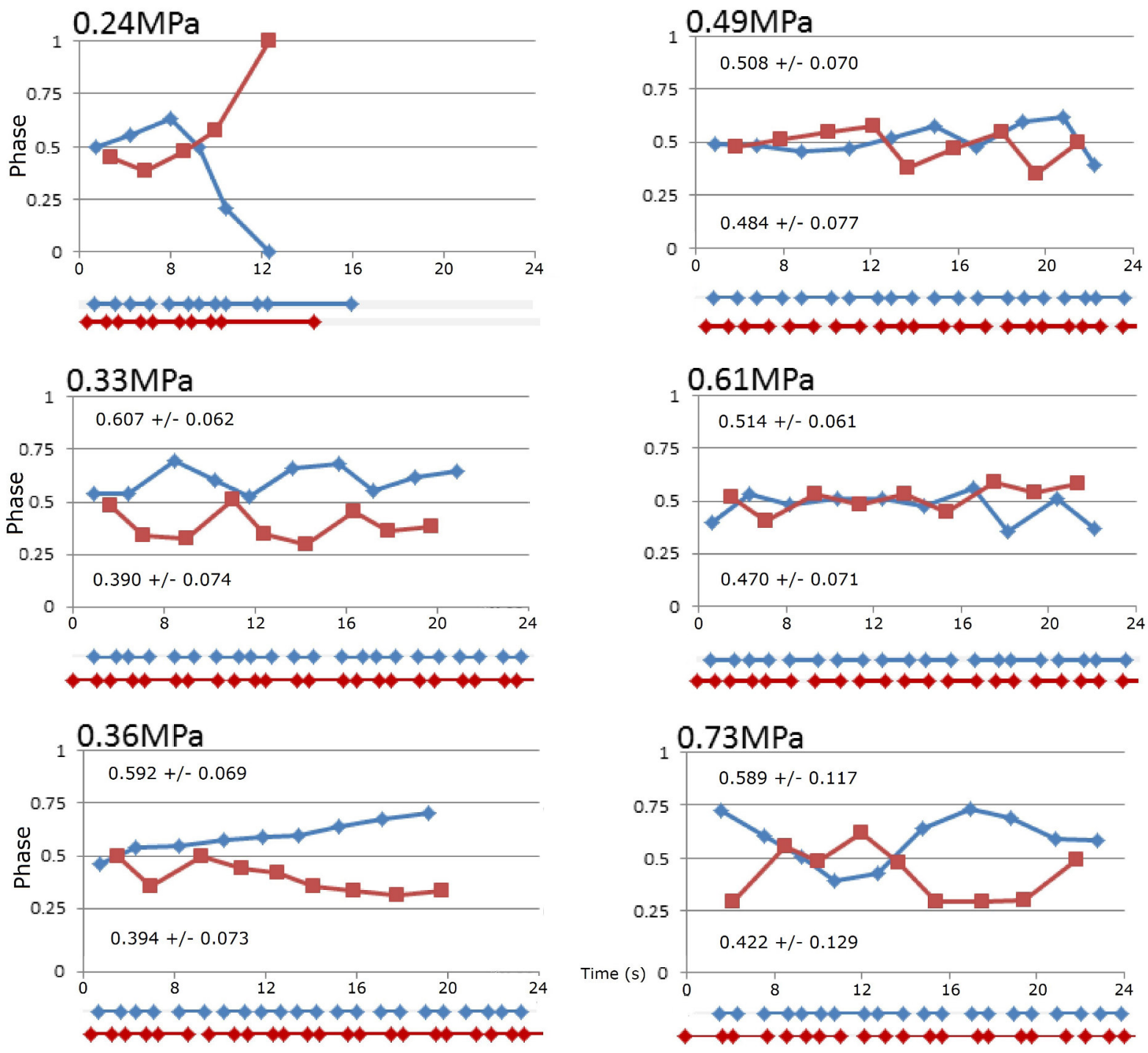

Fig. 12. Contribution to alternating gait for different values of rectus femoris pressure during stance. Red lines represent right hindlimbs, blue lines represent left hindlimbs and their mean and standard deviation are depicted on each graph.

In Fig. 12 rectus femoris contribution to stability was measured. Although a lower limit for stability could be observed, there was no maximum pressure which could degrade walking stability. All experiments with pressure below $0.3 \mathrm{MPa}$ eventually collapsed the robot for lack of knee joint stiffness, where the muscle vastus lateralis was eventually trying to keep the robot standing on its own. Best stability results were found with muscle pressure between $0.49 \mathrm{MPa}$ and $0.61 \mathrm{MPa}$, where we assume that an optimal knee compliance was achieved.

\subsection{Obstacle influence on alternating gait}

Changing the walking environment has a negative effect on biological gait, which is shortening the swing/touch down cycle. In Figures 13 and 14 the hindlimb passes through a $17.5 \mathrm{~mm}$ obstacle and hip, knee and ankle joints angles are registered during the

\footnotetext{
This is an offprint and may differ from the original version.
}

experiment. As seen on Fig. 13 the first contact with the obstacle happens while leaving lift off phase to swing, "kicking" the obstacle (marked with (a) in Fig. 14). This disturbance affects the knee and ankle angles, generating an abnormal swing phase, and has no effect on hip angle (this first disturbance is represented as the first half of the period marked as obstacle in Fig. 14). Upon touching the obstacle instead of the floor, the joints have to compensate the height difference with the muscular compliance provided by the artificial muscles. During this condition, the ankle which was usually in phase with the other 2 joints bends faster upon touching the obstacle (b), slipping and touching it for a second time (c), reaching a stance position with abnormal behavior and proceeding to swing again (last spike inside obstacle mark).

The same disturbances affect the knee joint in a different way. While "kicking" the obstacle, knee joints behave as if angles were offset in $-10^{\circ}$, decreasing as a whole during the first half of the 


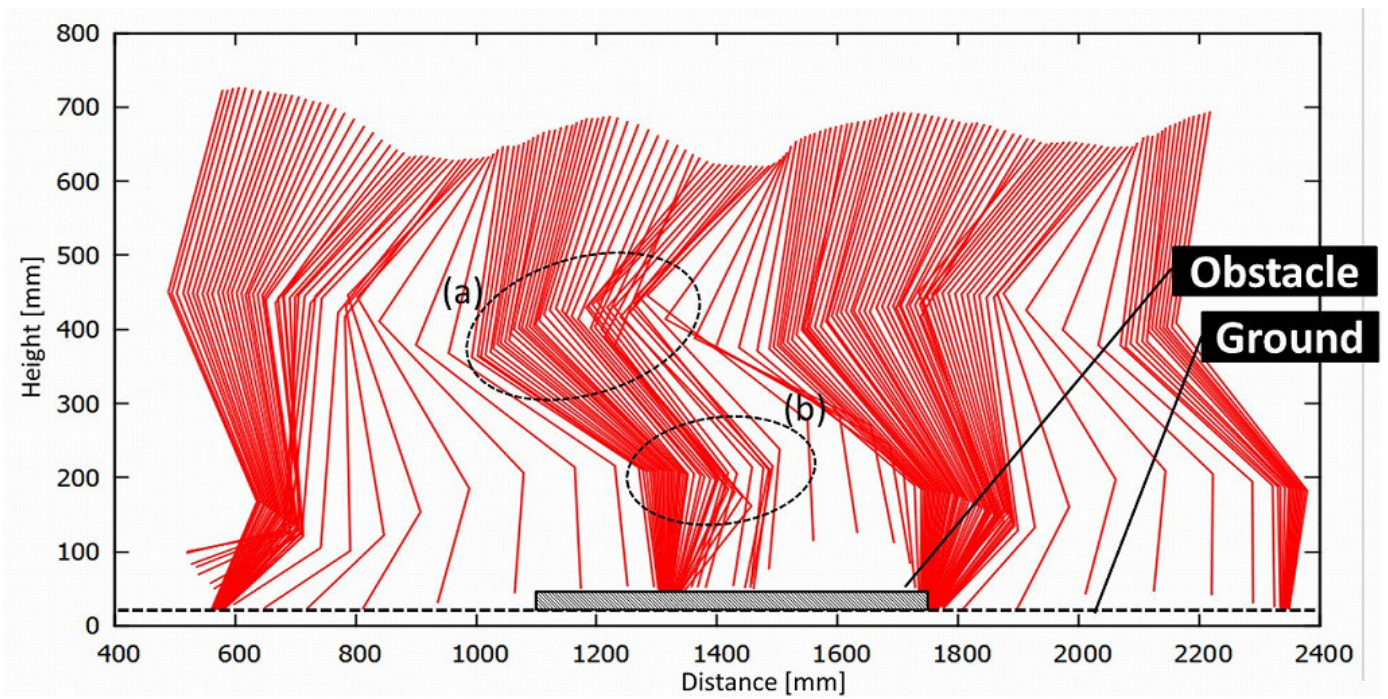

Fig. 13. Influence of a $17.5 \mathrm{~mm}$ obstacle on the uncoupled alternating gait behavior. Upon finishing touch down phase prematurely, knee (a) and ankle (b) joints react differently to keep stability.

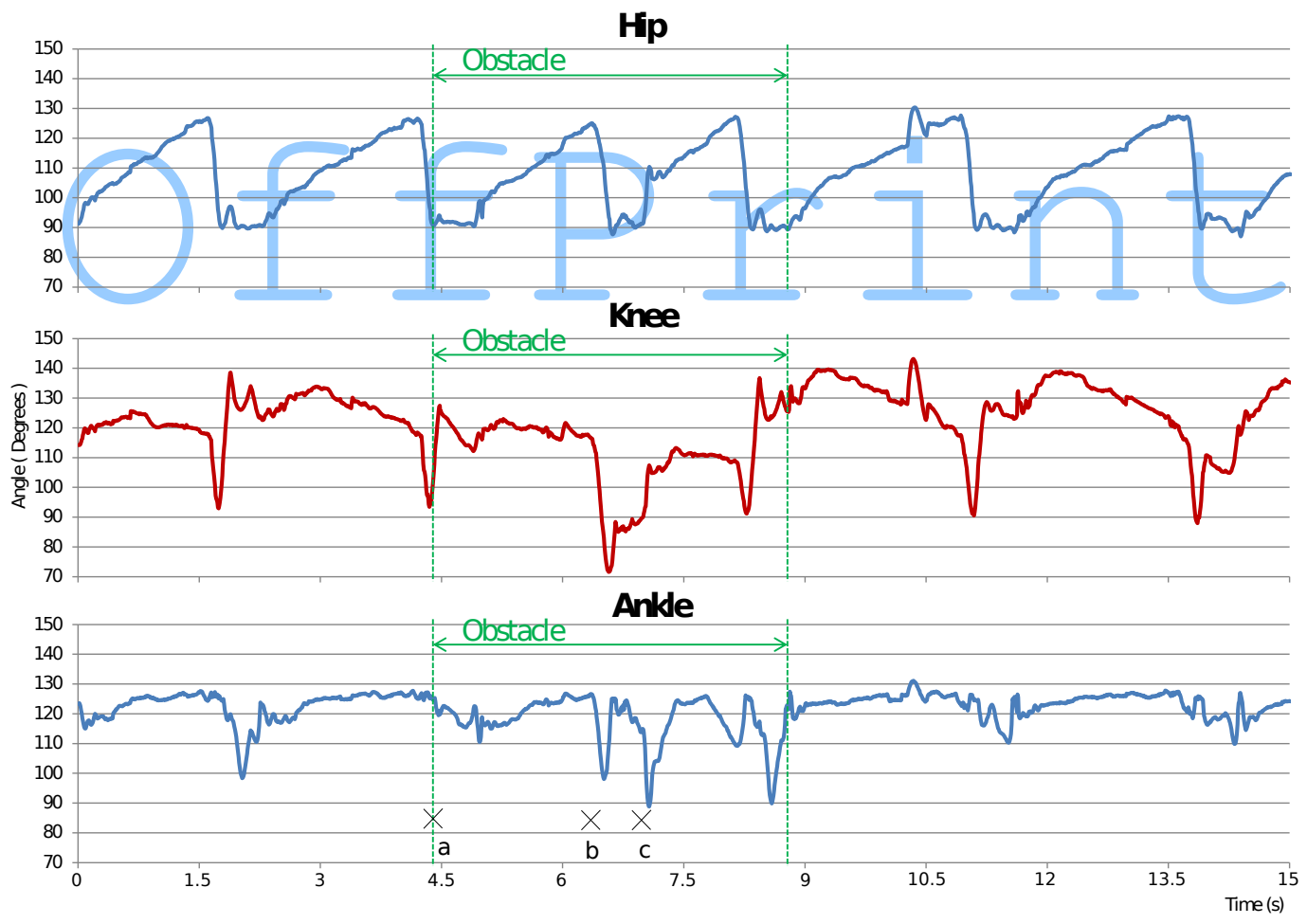

Fig. 14. Influence on hip, knee and ankle joint angles of a $17.5 \mathrm{~mm}$ obstacle on the uncoupled alternating gait behavior.

obstacle mark in Fig. 14. In the second half, where slippage and stance in obstacle happens, knee angles are offset even more to compensate for the different height, returning to normal behavior right after the obstacle is passed. Similarly to observations from gastrocnemius/rectus femoris muscles, the knee joint seems more stable than the ankle joint, being the hip joint the least susceptible to disturbances of all. While distal joints are more prone to high frequency disturbances, proximal joints only feel low frequency one, being the knee in the medium frequency range.

The presence of a strong state attractor on the unloading rule was enough to overcome obstacles on the treadmill, as shown in Fig. 15. Nevertheless, obstacles with $35 \mathrm{~mm}$ and beyond could not 

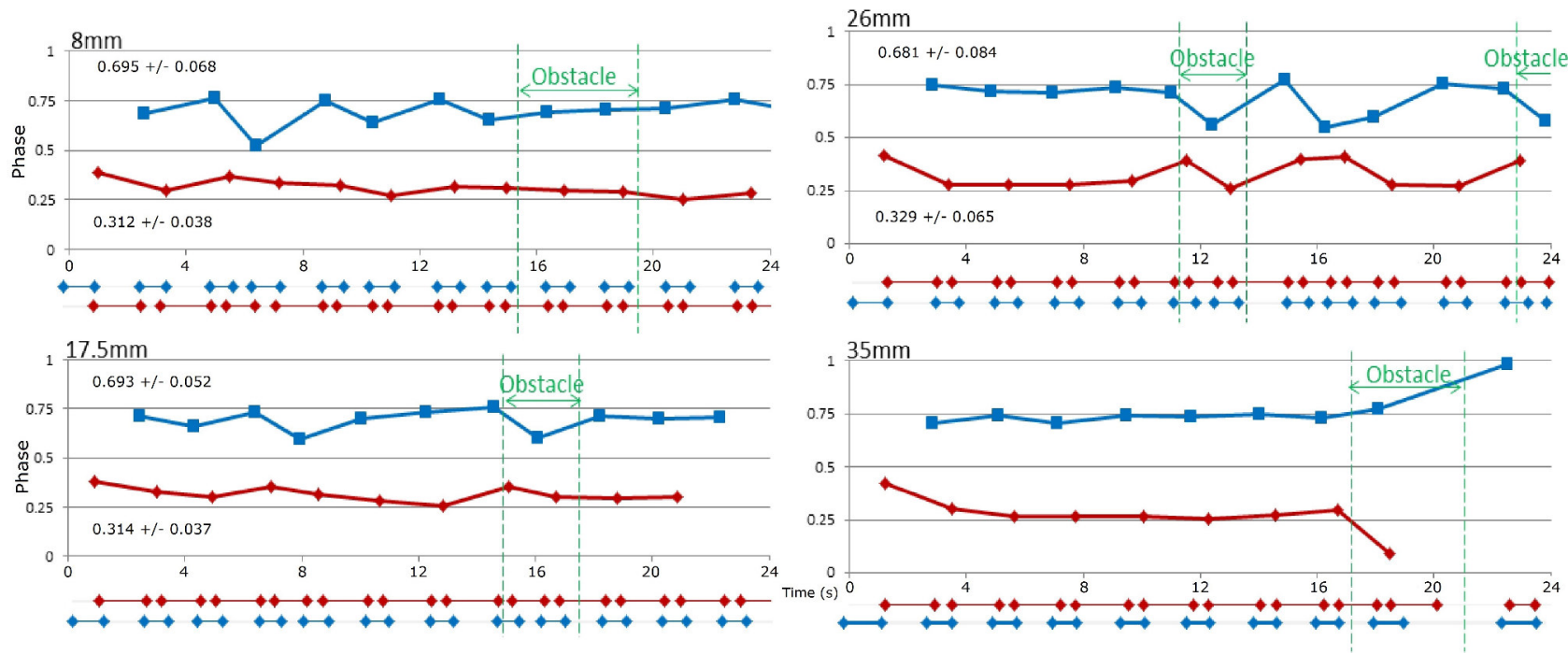

Fig. 15. Trials with different obstacles, probing Pneupard's limits overcoming disturbances. Red lines represent right hindlimbs, blue lines represent left hindlimbs and their mean and standard deviation are depicted on each graph.

be cleared with this simple rule. When passing through smaller obstacles, the transition from swing to touch down phase occur in midair, cancelling leg flexion forces and preparing the leg for landing, where sensory feedback will start the stance phase. We believe that there were mainly two falling conditions:

(1) Although the floor clearance from the robot is $40 \mathrm{~mm}$, this value is only true at the middle of the swing phase, with smaller values in the beginning and end of swing. This condition results in obvious consequences, with the robot stumbling on the obstacle, with a possible solution being an improved ground clearance.

(2) The negative influence on the opposite leg when the hindlimb touches the obstacle without transitioning from swing to touch down phases. This condition is less obvious, and better explained by the unloading rule itself: When the ground reaction force decreases below a threshold, the hindlimb finishes stance phase. This means that when an unprepared hindlimb touches the floor and does not enter stance phase, the small transfer of ground reaction force to this hindlimb may accelerate the opposite hindlimb transition, resulting in both limbs at swing at the same time.

\subsection{Study limitations}

The construction of this biomimetic platform aimed to explore locomotion as seen in real animals, and two limitations have to be observed:

-Pneumatic muscles are by far the best representation in real life robots to biological muscles. Researches with hydraulic, electric motors, intelligent plastics and even bio-cultivated muscles could not produce actuators with better power-to-weight ratio, long use reliability or even price. Nevertheless, pneumatic muscles are not real muscles, and their behaviors are not exactly the same, although closer than other options.

-Unloading rule in animals use muscular unloading, which takes into consideration not only ground reaction forces, but also variating moment arms at the ankle joint. Our approach only consid- ered the former, not fully representing the biomechanical reality. Even though such approach may be considered as a simplification of the biological structure, it was faithful enough to reproduce observed phenomena. Further studies will take into account the variable moment arm at the ankle by measuring the force between muscle and structure.

\section{CONCLUSION}

In this work we investigated the alternating gait of feline uncoupled hindlimbs through the unloading rule. Using Pneupard as a test platform, we tried to physically reproduce biological phenomena hitherto hypothesized by biologists and simulated by scientists.

Initially, the design process for the biomimetic robot is explained, considering limb dimensions, important muscles and realistic moment arms. Comparisons between biological and artificial muscles are established and the EMG-based muscular activation pattern is presented. A finite state control is proposed, using the unloading of ground reaction force to transit from stance to lift off phase, having no coupling between right and left hindlimb. Before experiments it is verified that the coupling between hindlimbs is not needed and Pneupard is capable of a stable alternating gait on the treadmill with this simple biological rule.

Experiments analyze the contribution of individual muscles (rectus femoris and gastrocnemius) to the alternating gait, and different force thresholds to perform stance-to-swing transition are verified. The ability to overcome obstacles with different heights is tested and the robot is capable of keeping alternating stability against different disturbances while powered by the unloading rule.

As future outcomes of this research we could mention a better understanding of felines, explaining decerebrate walking and muscular roles during stepping. A deeper knowledge on animal locomotion would finally allow us to replicate it. From a robotic side, reproducing animal locomotion, which surpasses robotic performance in so many different ways, would lead to highly adaptive behavior in legged robots, increasing the range of applications to robots in society. 


\section{REFERENCES}

Bertsekas DP, Tsitsiklis JN. Neuro-dynamic programming: an overview. In: Proc. Int. Conf. Decision and Control. 1995, p. 560-564.

Engberg I, Lundberg A. An electromyographic analysis of muscular activity in the hindlimb of the cat during unrestrained locomotion. Acta Physiol Scand. 1969;75:614-630.

Goslow Jr. GE, Reinking RM, Stuart DG. The cat step cycle: hind limb joint angles and muscle lengths during unrestrained locomotion. J Morphol. $1973 ; 141: 1-42$.

Prilutsky BI, Herzog W, Allinger TL. Mechanical power and work of cat soleus, gastrocnemius and plantaris muscle during locomotion: possible functional significance of muscle design and force patterns. J Exp Biol.1996;199:801-814.

Prilutsky BI, Herzog W, Leonard T. Transfer of mechanical energy between ankle and knee joints by gastrocnemius and plantaris muscles during cat locomotion. J Biomech. 1996;29:391-403.

Hudson PE, Wilson AM et al. Functional anatomy of the cheetah (Acinonyx Jubatus) hindlimb. J Anat. 2011;218:363-374.

Hudson PE, Wilson AM et al. Functional anatomy of the cheetah (Acinonyx Jubatus) forelimb. J Anat.2011;218:375-385.

Lam T, Pearson KG. The role of proprioceptive feedback in the regulation and adaptation of locomotor activity. In: Sensorimotor Control of Movement and Posture. New York: Kluwer Academic; 2002. p. 343-356.

Severin FV. The role of the gamma motor system in the activation of the extensor alpha motor neurones during controlled locomotion. Biophysics. 1970;15:1096-1102.

Hiebert GW, Pearson KG. Contribution of sensory feedback to the generation of extensor activity during walking in the decerebrate cat. J Neurophysiol. 1999;81:758-770.

Yakovenko S, Gritsenko V, Prochazka A. Contribution of stretch reflexes to locomotor control: a modeling study. Biol Cybern. 2004;90:146-155.

Ekeberg Ö, Pearson KG. Computer simulation of stepping in the hind legs of the cat: an examination of mechanisms regulating the stance-to-swing transition. J Neurophysiol. 2005;94:4256-4268.

Aschenbeck K, Kern N, Bachmann R, Quinn R. Design of a quadruped robot driven by air muscles. In: Proc. IEEE Int. Conf. Biomed. Robot. and Biomechatronics. 2006, p. 875-880.

Tsujita K, Kobayashi T, Inoura T, Masuda T. Gait transition by tuning muscle tones using pneumatic actuators in quadruped locomotion. In: Proc. IEEE Int. Conf. on Intelligent Robots and Systems. 2008, p. 2453-2458.

Lewisy M, Buntingy M, Salemi B, Hoffmann H. Toward ultra high speed locomotors: design and test of a cheetah robot hind limb. In: Proc. IEEE Int. Conf. on Robotics and Automation. 2011, p. 1990-1996.

Yamada Y, Kuniyoshi Y et al. Neural-body coupling for emergent locomotion: a musculoskeletal quadruped robot with spinobulbar model. In: Proc. IEEE Int. Conf. on Intelligent Robots and Systems. 2011, p. 14991506.

van Ingen Schenau GJ, Bobbert MF, Rozendal RH. The unique action of bi-articular muscles in complex movements. J Anat. 1987;155:1-5.

Maufroy C, Nishikawa T, Kimura H. Stable dynamic walking of a quadruped robot "kotetsu" using phase modulations based on leg loading/unloading. In: Proc. IEEE Int. Conf. on Intelligent Robots and Systems. 2010, p. 5225-5230.

Day L, Jayne B. Interspecific scaling of the morphology and posture of the limbs during the locomotion of cats (Felidae). J Exp Biol. 2007;210:642657.

Rosendo A, Narioka K, Hosoda K. Muscle roles on directional change during hopping of a biomimetic feline hindlimb. In: Proc. IEEE Int. Conf. on Robotics and Biomimetics. 2012, 6pp.
Pratt GA, Williamson MM. Series elastic actuators. In: Proc. IEEE Int. Conf. on Intelligent Robots and Systems. 1995, p. 399-406.

Hosoda K, Sakaguchi Y, Takayama H, Takuma T. Pneumatic-driven jumping robot with anthropomorphic muscular skeleton structure. Auton Robot. 2010;28:307-316.

Boblan I. A human-like robot hand and arm with fluidic muscles: biologically inspired construction and functionality. In: Embodied Artificial Intelligence. Dagstuhl Castle: Springer; 2004.

Klute G, Czerniecki J, Hannaford B. Artificial muscles: Actuators for biorobotic systems. Int. J. Robotics Res. 2002;21:295-309.

Xie SQ, Jamwal PK. An iterative fuzzy controller for pneumatic muscle driven rehabilitation robot. Expert Systems with Applications. 2011;38:8128-8137.

Azizi E, Roberts TJ. Variable gearing in a biologically inspired pneumatic actuator array. Bioinspir. Biomim. 2013;8: 8pp.

Pierotti D et al. Electromyographic activity of cat hindlimb flexors and extensors during locomotion at varying speeds and inclines. Brain Res. 1989;481:57-66.

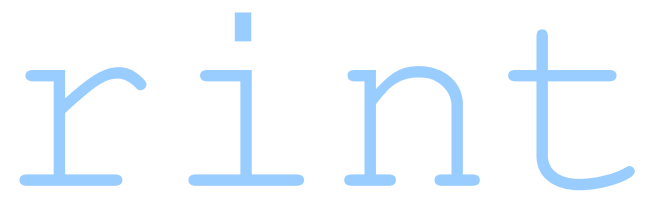

